\title{
REVISÃO DOS GÊNEROS Eurypodius GUÉRIN, 1825, Anomalothir MIERS, 1879 E Eucinetops STIMPSON, 1860, NAS COSTAS CARIBE E ATLÂNTICA DA AMÉRICA DO SUL (CRUSTACEA, DECAPODA, MAJIDAE)
}

Petrônio Alves COELHO

Departamento de Oceanografia da UFPE Bolsista $\mathrm{CNPq}$

\section{ABSTRACT \\ Revision of the genera Eurypodius Guérin, 1825, Anomalothir Miers, 1879 and Eucinetops Stimpson, 1860, in the Caribbean and Atlantic coasts of South America (Crustacea, Decapoda, Majidae).}

The genera Eurypodius, Anomalothir and Eucinetops are transferred from subfamily Inachinae, the first one to subfamily Eurypodiinae MacLeay, 1838 and the others to subfamily Anomalopodinae Stimpson, 1871. These genera are represented by the species E. latreillei Guérin, 1828, A. furcilatus (Stimpson, 1871) and E. blakciana Rathbun, respectively.

Key words: Decapoda, Majidae, Eurypodius, Anomalothir, Eucinetops.

\section{INTRODUÇÃO}

O estudo dos gêneros Eurypodius, Anomalothir e Eucinetops faz parte de uma pesquisa sobre os Majidae do litoral atlântico e caribe da América do Sul que será publicado por partes. Estes gêneros possuem uma história muito rica em acontecimentos, e apenas alguns momentos serão lembrados no presente estudo.

O primeiro gênero de Majidae descrito das costas atlântica e caribe da América do Sul foi Eurypodius, e a primeira espécie, Eurypodius latreillei, por GUÉRIN (1825, 1828). DANA (1851) designou Eurypodius o tipo da família Eurypodidae, constituída por este, juntamente com outros dois gêneros; MIERS (1879) colocou-o na subfamília Inachinae, pertencente à família Inachidae; ALCOCK (1895) classificou-o na aliança "Inachoida" da subfamília Inachinae; posteriormente, BALSS (1929) colocou-o entre os Macrocheiroidea, junto com outros gêneros primitivos de Majidae, porém no interior da subfamília Inachinae.

GRIFFIN (1966a) afirmou que Eurypodius possui grandes afinidades com Naxia Latreille, da Austrália e Nova Zelândia, porém outros autores colocam os dois em subfamílias distintas; por exemplo, este último faz parte da tribo Naxiini da subfamília Majinae de acordo com STEVCIC (1994). Uma espécie colocada por GRIFFIN (1966a) no gênero Naxia, a saber, Trichoplatus huttoni A. Milne Edwards, poderia ser relacionada a Eurypodius (vide GRIFFIN \& TRANTER, 1986). Segundo a opinião geral dos carcinólogos, Eurypodius, não possui afinidades com os demais gêneros de Majidae encontrados na área, devendo ser colocado numa unidade taxonômica separada.

O nome Anomalopus, que tinha sido proposto por STIMPSON (1871) para um gênero de Majidae, tendo sido empregado anteriormente para répteis, foi substituído por Anomalothir (MIERS, 1879), o qual vem sendo empregado desde então. STIMPSON (1871) havia proposto classifica-lo na subfamília Anomalopinae, erigida por ele mesmo; 
MIERS (1879), no entanto, o colocou entre os Acanthonychinae, o que foi aceito por RATHBUN (1894). ALCOCK (1895) o transferiu, com dúvida, para a subfamília Inachinae, na "aliança" Leptopodioida. A partir desta data, a maioria dos autores o tem colocado entre os Inachinae. Apenas em 1996 foi a espécie A. furcillatus referida para a área estudada (MELO, 1996), embora tenha sido citada anteriormente em dissertação de mestrado.

A. MILNE EDWARDS (1875) comparou Eucinetops Stimpson com Criocarcinus Milne Edwards e com Stenocionops Latreille, diferindo dos mesmos pelas órbitas, livres por baixo, e com Micippa Leach, do qual seria próximo pela forma geral. Desta forma, o gênero era classificado entre os Mithracinae (esta semelhança é tal que LEMOS DE CASTRO (1953) descreveu no gênero Eucinetops uma espécie que posteriormente se verificou ser um Mithracinae). MIERS (1879) transferiu Eucinetops para a subfamília Inachinae da família Inachidae, porém seu conceito de Inachinae inclui não só o gênero Inachus Weber, como a maioria do que é considerado aqui Inachoidinae, além do gênero Camposcia Latreille. MIERS (1879) comentou ainda que Eucinetops, pela forma da carapaça, olhos e maxilípedes teria alguma afinidade com Micippa e outros gêneros próximos, colocados por ele na família Maiidae, atualmente classificados como Mithracinae. ALCOCK (1895) incluiu este gênero na "aliança" Inachoida da subfamília Inachinae, porém com muita dúvida, acrescentando ainda que, como STIMPSON (1871) tinha sugerido, deveria estar próximo de Micippa. RATHBUN (1925) não teve qualquer dúvida em considera-lo como Inachinae, porém BALSS (1929) o transferiu para a subfamília Ophthalmiinae. GARTH (1958) não fez qualquer referência a esta controvérsia, e o admitiu entre os Inachinae sem qualquer discussão. GUINOT \& RICHER DE FORGES (1987) consideram que a sua classificação deveria ser revisada. Dando continuidade a esta sugestão, o gênero é separado da subfamília Inachinae e classificado entre os Anomalopinae. Uma espécie, E. blackiana Rathbun, que tinha sido assinalada para Bonaire por RATHBUN (1925, 1936) é referida no presente trabalho também para a Colômbia.

Em função destas e de muitas outras mudanças, a identificação dos Majidae, na costa leste da América do Sul, tornou-se tarefa reservada aos especialistas. Dado o interesse nos estudos ecológicos, fisiológicos, etológicos, etc., sobre estes braquiuros, tornou-se necessário realizar uma revisão da sua sistemática. Desta forma, o não especialista poder ter ao seu alcance uma obra permitindo efetuar suas identificações, incluindo não apenas redescrições das espécies encontradas até o presente momento, como dados disponíveis sobre alguns aspectos de sua biologia. Dado o volume do trabalho, foi resolvido publica-lo por partes.

\section{MATERIAL E MÉTODOS}

O material estudado foi coligido por expedições oceanográficas e coletas costeiras, encontrando-se depositado principalmente em seis instituições: Departamento de Oceanografia da Universidade Federal de Pernambuco (DOPE), Museu de Zoologia da Universidade de São Paulo (MZSP), Museu Nacional da Universidade Federal do Rio de Janeiro (MNRJ), Coleção Carcinológica da Fundação Universidade de Rio 
Grande (FURG), Zoologisches Institut und Museum, Universitat Hamburg (ZMH) e Forschungsinstitut und Naturmuseum Senckenberg (SMF).

As listas das expedições oceanográficas e das localidades costeiras são apresentadas em anexo, contendo número da estação ou local, data, latitude, longitude, profundidade e tipo de fundo; as expedições estão organizadas em ordem alfabética e as estações seguem ordem numérica crescente. As coletas costeiras estão arrumadas em ordem geográfica no sentido norte - sul, desde a Colômbia até a Argentina.

O trabalho apresenta, sob a forma de chave, uma classificação dos Majidae, baseada numa análise filogenética da família, a ser publicada oportunamente. Nesta chave não são levadas em conta as afinidades entre os Inachoidinae e a família Hymenosomatidae, apontadas por GUINOT \& RICHER DE FORGES (1997).

Para cada gênero são fornecidos: sinonímia, redescrição, espécie tipo e distribuição geográfica; para cada espécie são apresentados: sinonímia, localidade-tipo, outros registros, material examinado, distribuição geográfica, redescrição, dimensões, variações, coloração, observações e comentários. As sinonímias apresentadas para os gêneros e espécies são parciais, indicando apenas algumas das obras consideradas de maior importância. As localidades tipo estão referidas conforme a citação dos autores. A seção intitulada "outros registros" não tem a pretensão de incluir toda a literatura e está organizada em ordem geográfica de norte para sul. A distribuição geográfica foi baseada em informações bibliográficas (isto é, constitui uma das indicações existentes nas seções intituladas "localidade-tipo" e "outros registros") e em dados das coleções carcinológicas das instituições citadas anteriormente. As redescrições dos gêneros e das espécies incluem dados recolhidos na literatura e no exame de espécimes. Os comentários sobre as espécies se referem à sua classificação, enquanto as observações trazem indicações de ordem biológica. O conteúdo dos outros itens do texto incluem dados de literatura e observações pessoais.

\section{RESULTADOS}

Família MAJIDAE Samouelle, 1819

Redescriçãoo.- Carapaça estreitada anteriormente. Superfícies cobertas por pelos em forma de gancho. Antênulas dobradas longitudinalmente. Artículo basal das antenas fundido ao epístoma e, freqüentemente, à fronte. Quelípedes muito móveis, não havendo diferenças em o direito e o esquerdo, projetando-se muito além das margens ântero-laterais da carapaça, dedos pouco encurvados. Suturas do esterno desde a IV/V até VII-VIII, não interrompidas.

Comentários.- Os majídeos apresentam uma diversidade de forma muito grande. Algumas estruturas têm sido empregadas como caracteres sistemáticos: tamanho e forma do rostro; espinhos e tubérculos da superfície dorsal da carapaça e das margens laterais; morfologia do septo interantenular; morfologia do segmento basal das antenas; tamanho do pedúnculo das antenas e a possibilidade vê-los dorsalmente; estrutura das órbitas; comprimento dos quelípedes e patas ambulatórias. Em função

Trab. Oceanog. Univ. Fed. PE, Recife, 27(1):149-168, 1999. 
desta variabilidade, a família sofreu grandes revisões, em escala mundial, que mudaram completamente o esquema de classificação, sendo aqui revistos alguns momentos considerados importantes.

DANA (1851) dividiu os caranguejos que aqui são considerados como uma única família, reconhecendo um total de cinco famílias (Majidae, Tychidae, Eurypodidae, Leptopodidae e Periceridae) e vinte e sete subfamílias.

MIERS (1879) julgou ter motivos para repartir os Majidae em três famílias (Inachidae, Maiidae e Periceridae) e onze subfamílias.

ALCOCK (1895) reuniu todas estas famílias numa só, porém dividida em quatro subfamílias: Maiinae, Pisinae, Acanthonychinae e Inachinae. Este sistema de classificação teve vários seguidores. Entre estes, alguns dos mais conhecidos foram: RATHBUN (1925), em seu estudo monográfico sobre os Oxyrhyncha das Américas; BOUVIER (1940), no trabalho sobre a fauna da França; CAPART (1951) e MONOD (1956), em estudos sobre a fauna da África Ocidental e GUINOT (1966) no catálogo dos braquiúros do Oceano Índico Ocidental e do Mar Vermelho. BARNARD (1950), no catálogo dos decápodos da África do Sul, empregou sistema semelhante, porém elevando as subfamílias à famílias, e substituindo os nomes Pisinae e Majinae por, respectivamente, Blastidae e Mamaidae.

BALSS (1929), igualmente, considerou uma única família, porém dividida em nove subfamílias: Majinae, Mithracinae, Macrocoelominae, Ophthalmiinae, Pisinae, Hyasteninae, Acanthonychinae, Camposcoidea e Macrocheiroidea. BENNET (1964) seguiu o sistema de BALSS (1929) em estudo sobre os majídeos da Nova Zelândia.

Num estudo monográfico sobre os Majidae do Pacífico Oriental, GARTH (1958) modificou a classificação de BALSS (1929) nos seguintes pontos: a) reuniu os Mithracinae e Macrocoelominae para constituir a subfamília Mithracinae; b) incluiu os Pisinae e os Hyasteninae numa única subfamília, Pisinae; c) restabeleceu a subfamília Inachinae, agrupando os Macrocheiroidea e os Camposcoidea; d) erigiu uma nova subfamília, Oregoniinae. Desta forma, a família Majidae ficou subdividida em Oregoniinae, Inachinae, Ophthalmiinae, Acanthonychinae, Pisinae, Majinae e Mithracinae. BOSCHI (1964), WILLIAMS (1965, 1984), GRIFFIN (1966a, b), SAKAY (1976), RODRÍGUEZ (1980) e MELO (1996), entre muitos outros, adotaram o sistema de BALSS (1929) modificado por GARTH. É interessante observar que, depois, BALSS (1957) abandonou seu próprio esquema de classificação de 1929 e adotou integralmente o de ALCOCK (1895).

Mais recentemente, GUINOT (1978) elevou a família Majidae à superfamília e, em consequência, as subfamílias tal como definidas por GARTH (1958), passaram ao nível de famílias. Posteriormente, DRACH \& GUINOT (1982) acrescentaram mais uma família, Inachoididae.

Finalmente, STEVCIC (1994) encontrou elementos para fundamentar uma divisão da família nas subfamílias Majinae, Pliosominae, Mithracinae, Planoterginae, Tychinae, Pisinae, Eurynolambrinae, Epialtinae e Inachinae; basicamente, esta é a classificação adotada no presente trabalho. Nos esquemas anteriores, os Inachinae são colocados no começo do esquema e os Majinae no fim, pois os autores consideram que as órbitas simples dos Inachinae são primitivas e as órbitas completas dos Majinae, 
derivadas. A classificação apresentada a seguir não leva em conta subfamílias não encontradas na área estudada, referindo-se apenas a Mithracinae, Tychinae, Pisinae, Epialtinae, Eurypodiinae, Anomalopodinae, Inachinae e Inachoidinae

Chave para identificação das subfamílias

1 - Olhos com órbitas completas ou quase, ornadas pelo artículo basal das antenas (que é largo, expandido de maneira a formar a porção inferior da órbita), pelo capuz supra-orbital e pelo lobo pós-orbital; espinho intercalado presente ou ausente Mithracinae

1'- Olhos sem órbitas ou com órbitas incompletas; pedúnculos oculares longos ou curtos

2 - Órbitas incompletas; pedúnculos oculares longos, apenas parcialmente protegidos por cima pelo supra-orbital em forma de espinho, protegidos ou não por baixo. Tychinae

2'- Órbitas incompletas ou ausentes; pedúnculos oculares curtos. 3

3 - Órbitas incompletas, ornadas pelo espinho supra-orbital e por um processo pósorbital no qual os olhos podem se ocultar.

..Pisinae

3'- Órbitas incompletas ou ausentes, porém desprovidas de processo pós-orbital no qual os olhos possam se ocultar

4 - Órbitas incompletas; olhos pouco móveis, em alguns casos ocultos por cima pelo espinho supra-orbital, noutros afundando ao lado do rostro; antenas com artículo basal em forma de triângulo truncado Epialtinae

4'- Órbitas ausentes; pedúnculos oculares geralmente longos, em alguns casos não retráteis, noutros retráteis contra os lados da carapaça, ou então há um espinho pósocular incapaz de oculta-los; artículo basal das antenas longo e delgado...... .5

5 - Um espinho intercalado situado entre os espinhos pré-ocular e pósocular...... Eurypodiinae

5'- Espinho intercalado ausente. ...6

6 - Machos e fêmeas com sete segmentos abdominais; rostro

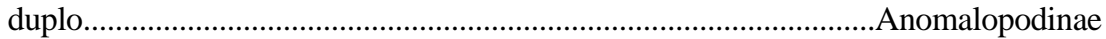

6'- Machos com seis segmentos e fêmeas com sete segmentos abdominais; rostro duplo ou simples. 7

7 - Margens póstero-laterais da carapaça desprovidas de placas; dentes pós-oculares pequenos, distantes dos olhos Inachinae

7'- Presença de placas visíveis dorsalmente ao lado das margens póstero-laterais da carapaça; dentes pós-oculares bem desenvolvidos. .Inachoidinae

\section{Subfamília EURYPODIINAE Mac Leay, 1838}

Redescrição.- Olhos sem órbitas; pedúnculos oculares longos; espinhos intercalado e pós-orbital presentes; artículo basal das antenas delgado; rostro duplo, 
constituído por dois espinhos contíguos; abdome constituido por sete elementos em ambos os sexos. Patas ambulatórias geralmente longas.

Comentários.- A subfamília compreende, na área estudada, apenas um gênero, Eurypodius, e uma espécie, E. latreillei. Não está incluído no escopo do presente trabalho uma listagem dos gêneros pertencentes a esta subfamília, caracterizada, principalmente, pela presença de espinho intercalado nas órbitas e pela morfologia das patas ambulatórias.

\section{Gênero Eurypodius Guérin, 1825}

Eurypodius GUÉRIN, 1825:700.- RATHBUN, 1925:80.- GARTH, 1958:39.

Redescrição.- Carapaça alongado-triangular, moderadamente convexa, e com espinhos ou tubérculos na face dorsal. Rostro constituído por espinhos contíguos, pelo menos em parte de seu comprimento. Dente pós-ocular distinto, bem desenvolvido, porém dente pré-ocular ausente. Antenas visíveis ao lado do rostro. Mero dos maxilípedes externos truncado, o palpo se fixando em seu ângulo ântero-interno. Patas ambulatórias consideravelmente longas, com os própodos mais ou menos dilatados e comprimidos; dáctilos fracamente arqueados, capazes de se dobrar sobre a margem inferior dos própodos.

Espécie-tipo.- Eurypodius latreillei Guérin, 1828.

Distribuição geográfica.- Extremidade sul da América do Sul até o Peru, no Pacífico e até o Brasil, no Atlântico. Constituído por duas espécies: E. longirostris Miers no Pacífico e E. latreillei Guérin no Atlântico e no Pacífico.

\section{Eurypodius latreillei Guérin, 1828}

(Fig. 1)

Eurypodius latreillii GUÉRIN, 1828:354.- RATHBUN, 1925: 80, pls. 30, 31 e 215. 1996:182.

Eurypodius latreillei - GARTH, 1958:40, pl. B, fig. 1 e pl. 2, fig. 1.- MELO,

Localidade tipo.- Ilhas Malvinas.

Outros registros.- BRASIL: Rio de Janeiro (BELL, 1835; BORRADAILE, 1916), Rio Grande do Sul (MELO, 1990); ARGENTINA: Buenos Aires (BOSCHI, 1964; COELHO \& RAMOS, 1972), Chubut (RATHBUN, 1898, 1925; DOFLEIN \& BALSS, 1912; BOSCHI, 1964; MELO, 1990), Santa Cruz (RATHBUN, 1898, 1925; A. MILNE EDWARDS \& BOUVIER, 1923; BOSCHI, 1964; MELO, 1990), Terra do Fogo (DANA, 1851, 1852; MIERS, 1886; DOFLEIN \& BALSS, 1912; BOSCHI, 1964); MALVINAS (CUNNINGHAM, 1871; LENZ, 1902; LAGERBERG, 1905; DOFLEIN \& BALSS, 1912); BANCO BURDWOOD (LAGERBERG, 1905); CHILE (DANA, 1851, 1852; GARTH, 1957; BOSCHI, 1964); PERU (GARTH, 1958).

Material examinado.- Mais de 200 espécimes provenientes de 85 estações:

URUGUAI.- WB\#1873, 2 F (OV), MZSP.

BUENOS AIRES.- AS\#1440, 1 J, MZSP; AS\#2887, 1 F (OV), MZSP; AS\#2286, 1 F (OV), DOPE; WH\#690, 2 M, 1 F (OV), ZMH; WH\#693, 2 M, ZMH; 
WH\#499, 6 M, 1 F (OV), ZMH; WH\#697, 2 M, 2 F (OV), ZMH; WH\#696, 1 F (OV), ZMH; WH\#691, numerosos, ZMH; WH\#500, 1 M, 2 F (OV), ZMH.

RIO NEGRO.- WH683, 1M, ZMH; WH\#684, 2 F (OV), ZMH; WH\#508, 1 J, ZMH; WH\#509, 1 M, 1 F (OV), ZMH; WH\#510, 2M, ZMH; WH\#678, 2M, 1 F (OV), $\mathrm{ZMH}$; WH\#679, 2M, 2F (OV), ZMH.

CHUBUT.- WH\#681, 1 M, 1 F (OV), ZMH; WH\#520, 2M, 1 F (OV), ZMH; WH\#682, 3 F (OV), ZMH; WH\#521, 2M, 2 F(OV), ZMH; WH\#522, 1 F (OV), ZMH; WH\#671, 1 M, ZMH; WH\#675, 1 M, 2 F (OV), ZMH; WH\#674, 1 M, 1 F (OV), ZMH; WH\#673, 1 M, ZMH; Ostpatagonische Bank (a), 1 F, 2 J, ZMH; WH\#667, 1 M, ZMH; WH\#528, numerosos, ZMH; WH\#662, 1 M, ZMH; WH\#526, 5M, ZMH; Patagonische Bank, 2 M, 1 F, ZMH; Ostpatagonische Bank (b), 1 M, ZMH; WH\#661, 2 M, 2 F (OV), ZMH; WH\#660 2 M, 2 F (OV), ZMH; Comodoro Rivadavia, 1 M, 2 F, MNRJ.

SANTA CRUZ.- WH\#651, $1 \mathrm{M}, 1 \mathrm{~F}(\mathrm{OV}), \mathrm{ZMH}$; WH\#652, 4 F (OV), ZMH; WH \#534, 1 M, 2 F (OV), ZMH; WH\#537, 2 M, 1 F (OV), ZMH; WH\#536, 2 M, 2 F (OV), ZMH; WH\#655, 2 M, ZMH; WH\#656, 1 M, ZMH; WH\# 654, 2 F (OV), ZMH; Puerto Deseado, $1 \mathrm{~J}$, DOPE; WH\#546, 4 M, 1 F (OV), ZMH; WH\#545, $1 \mathrm{~F}$ (OV), ZMH; WH\#641, 2 F, ZMH; WH\#643, 3 F (OV), ZMH; WH\#642, 1 M, ZMH; WH\#644, 1 M, ZMH; WH\#640, 2 M, ZMH; WH\#639, 1 M, 1 F (OV), ZMH; WH\#633, 2 M, 1 F (OV), ZMH; WH\#630, 2 F (OV), ZMH; WH\#628, 3 M, ZMH; WH\#627, 2 F (OV), ZMH; WH\#629, 1 M, ZMH; WH \#632, 2 F (OV), ZMH; WH\#626, 1 F (OV), ZMH; WH\#631, 1 M, ZMH; WH\#623, 1 M, 2 J, ZMH; WH\#565, 1 F (OV), ZMH; WH\#568, 2 M, 2 F (OV), ZMH; WH\#578, 1 F, ZMH; WH\#619, 1 M, 1 F (OV), ZMH; WH\#621, 2 M, ZMH; WH\#617, 1 M, 2 F (OV), ZMH; WH \#616, 4 F (OV), ZMH; WH\#620, 1 F (OV), ZMH; WH\#618, 2 M, 1 F (OV), ZMH; WH \#615, 1 F (OV), $\mathrm{ZMH}$; sem nome de localidade, $3 \mathrm{M}$, ZMH; WH\#607, $2 \mathrm{M}$, ZMH.

TERRA DO FOGO.- WH\#609, 3 M, ZMH; Sarmiento Bank, $2 \mathrm{M}, \mathrm{ZMH}$; WH\#606, 1 F (OV), ZMH; WH\#604, 1 M, ZMH; WH\#603, 1 M, 1 F (OV), ZMH; WH\#600, 2 M, ZMH; WH\#593, 1 M, ZMH.

ILHAS MALVINAS.- WH\#569, 3 M, ZMH; WH\#614, 1 F (OV), ZMH; Port Stanley, 2 M, 1 F, ZMH; Port Williams, 1 M, ZMH; WH\#580, 1 F (OV), ZMH; WH\#613, 1 M, ZMH.

BANCO BURDWOOD.- WH\#596, $1 \mathrm{~F}(\mathrm{OV}), \mathrm{ZMH}$; WH\#587, $3 \mathrm{M}, 2 \mathrm{~F}(1$ OV), ZMH; WH\#588, 1 M, ZMH; WH\#586, 1 M, FRAG., ZMH; WH\#582, 2 F (OV), ZMH; WH \#584, 1 M, ZMH; WH\#592, 1 M, ZMH.

Distribuição geográfica.- Attântico Ocidental: desde o Uruguai $\left(35^{\circ} \mathrm{S}\right)$ até o sul da América do Sul, ocasionalmente mais ao norte, no Rio de Janeiro; Pacífico Oriental: desde o sul da América do Sul até o Peru $\left(14^{\circ} \mathrm{S}\right)$.

Redescrição.- Carapaça triangular, pubescente, de superfície irregular e com vários espinhos.

Rostro bífido; espinhos longos, justapostos, quase horizontais.

Linha mediana da carapaça com cinco espinhos principais: dois gástricos, um genital, um cardíaco e outro na margem posterior da carapaça. Regiões branquiais com um espinho dorsal e uma fileira marginal de espinhos. 
Olhos sem órbitas; espinho intercalado saliente, seguido pelo espinho pósocular, mais desenvolvido e curvado para a frente. Pedúnculos oculares curtos e fortes.

Antenas longas, de comprimento quase igual ao da metade da carapaça; artículo basal seguido por dois tubérculos infra-oculares.

Quelípedes fortes. Patas ambulatórias e quelípedes com fileiras de pelos nas margens dorsal e ventral. Própodo das patas ambulatórias laminar, alargado, dáctilo encurvado, capaz de se dobrar sobre a margem ventral do própodo.

Machos e fêmeas com todos os segmentos abdominais livres.

Dimensões.- Atinge até cerca de $70 \mathrm{~mm}$ de comprimento.

Variações.- BOSCHI (1964) comentou a variabilidade da espécie, causa de uma extensa sinonímia. A largura da carapaça, a disposição dos espinhos e tubérculos, a dimensão e a forma do rostro, bem como o número e a disposição das cerdas estão sujeitos a variações não só ligadas ao sexo e à idade, como também há variações entre indivíduos do mesmo sexo e do mesmo tamanho.

Ecologia.- Segundo GARTH (1957, 1958) e BOSCHI (1964) a espécie vive desde o limite da baixa -mar até 142 metros de profundidade, penetrando em estuários da região patagônica, tanto na Argentina como no Chile. O material examinado foi coletado desde pequena profundidade até 1.040 metros, geralmente a partir de $80 \mathrm{~m}$, em salinidades que variaram entre 33,0 e $36,6 \%$ e temperatura entre 2,7 e $14,0^{\circ} \mathrm{C}$, podendo ser classificada, portanto, como espécie euribata de águas temperadas.

Observações.- Geralmente com o corpo recoberto por esponjas, hidróides, ascídias, briozoários e algas.

Comentários.- BELL (1835) referiu-se a material desta espécie que teria sido coletado no Rio de Janeiro por D. Miller, porém é provável que a amostra tenha sido recolhida no Pacífico Oriental, como o restante dos espécimens fornecidos por este coletor e mencionados naquele trabalho. A amostra citada por MELO (1990) foi obtida na estação WB\#1873, realizada no Uruguai, não valendo a referência para o Rio Grande do Sul. A citação de BORRADAILE (1916) da ocorrência da espécie no Brasil é considerada válida, não havendo no texto qualquer indicação da possibilidade da espécie ter sido coletada noutra localidade qualquer.

\section{Subfamília ANOMALOPODINAE Stimpson, 1871}

Redescrição.- Olhos sem órbitas; pedúnculos oculares não retráteis; artículo basal das antenas delgado; carapaça alongada; rostro bífido; abdome constituido por sete elementos em ambos os sexos. Patas ambulatórias geralmente longas e delgadas.

Comentários.- Da mesma forma que os Inachinae e Inachoidinae, os Anomalopodinae diferem dos Eurypodiinae pela ausência de espinho intercalado, porém se destacam dos Inachinae e dos Inachoidinae pelo formato da carapaça e das patas ambulatórias e, principalmente, pelo abdome semelhante ao dos Eurypodiinae, ou seja, constituído por sete elementos em ambos os sexos. Não faz parte do escopo do presente trabalho a discussão sobre os gêneros existentes noutras áreas que possam ser 
classificados nesta subfamília, porém nela estão incluídos os gêneros Anomalothir e Eucinetops.

Chave para identificação dos gêneros

1 - Rostro longo (comprimento igual ou superior ao comprimento pós-ocular); patas ambulatórias do primeiro par alongadas Anomalothir 1'- Rostro e patas ambulatórias curtos. Eucinetops

Gênero Anomalothir Miers, 1879

Anomalopus STIMPSON, 1871:124 (nome pré-ocupado).- A. MILNE EDWARDS, 1879:187.

Anomalothir MIERS, 1879:648.- RATHBUN, 1925:23.-GARTH, 1958:48.

Redescrição.- Carapaça muito alongada, quase subcilíndrica; rostro longo, delgado, bífido. Olhos sem órbitas; espinho pré-ocular pequeno e agudo; espinho pósocular um pouco menor. Antenas visíveis dorsalmente; artículo basal estreito. Fossetas antenulares grandes. Epístoma de comprimento igual a 2/3 da largura.

Mero dos maxilípedes externos sem qualquer entalhe para inserção do palpo; ângulo externo saliente. Quelípedes na fêmea mais curtos que a carapaça, porém mais longos no macho. Patas ambulatórias do primeiro par de comprimento igual ao duplo do da carapaça, com o dáctilo quase retilíneo e igual a 3/4 do própodo. Patas ambulatórias dos dois pares posteriores mais curtas e mais fortes que as dos dois pares anteriores, e com extremidade preênsil, as do penúltimo par mais curtas que as do último.

Espécie-tipo.- Anomalopus furcillatus Stimpson, 1871.

Distribuição geográfica.- Ocorre no Atlântico Ocidental, desde a Carolina do Norte até o Rio Grande do Sul e no Pacífico Oriental, em Galápagos. Constituído por três espécies: A. frontalis (A. Milne Edwards) e A. furcillatus (Stimpson) no Atlântico e A. hoodensis Garth, no Pacífico.

\section{Anomalothir furcillatus (Stimpson, 1871)}

(Fig. 2)

Anomalopus furcillatus STIMPSON, 1871:125.- A. MILNE EDWARDS, 1879: 188, pl. 35, figs. 4-4d.

Anomalothir furcillatus - MIERS, 1879:648.- A. MILNE EDWARDS \& BOUVIER, 1923:368.- RATHBUN, 1925:24, pl. 9, fig. 2 e pl. 206.- WILLIAMS, 1965:236; 1984:294.- POWERS, 1977: 43. MELO, 1996:181.

Localidade tipo.- "The Samboes", Flórida.

Outros registros.- ESTADOS UNIDOS: Carolina do Norte (RATHBUN, 1925), Flórida (A. MILNE EDWARDS, 1879; A. MILNE EDWARDS \& BOUVIER, 1923; RATHBUN, 1925; ABELE \& KIM, 1986); CUBA (A. MILNE EDWARDS, 1879; RATHBUN, 1925); MÉXICO (A. MILNE EDWARDS, 1879; A. MILNE EDWARDS \& BOUVIER, 1923; RATHBUN, 1925); JAMAICA (RATHBUN, 1925); SANTA CRUZ (A. MILNE EDWARDS \& BOUVIER, 1923; RATHBUN, 1925); DOMINICA (A. MILNE EDWARDS \& BOUVIER, 1923); GUADALUPE 
(RATHBUN, 1925); S. VICENTE (A. MILNE EDWARDS \& BOUVIER, 1923); GRANADA (A. MILNE EDWARDS \& BBOUVIER, 1923; RATHBUN, 1925); BRASIL: Rio Grande do Sul (MELO, 1996; SOUZA, 1997).

Material examinado.- cinco espécimes provenientes de quatro estações:

RIO GRANDE DO SUL.- AT\#8.16, 1M, FURG; WB\#437, 1 M, MZSP; WB\#1856, 2 F (OV), MZSP; WB\#458, 1 F (OV), MZSP.

Distribuição geográfica.- Atlântico Ocidental: desde a Carolina do Sul até Granada; Rio Grande do Sul.

Redescrição.- Carapaça muito alongada, quase subcilíndrica, pubescente, com quatro tubérculos na região gástrica, um na cardíaca, um na intestinal e um em cada região branquial. Margens laterais com uma fileira de grânulos, continuando na região pterigostomiana. Regiões hepáticas encimadas por grânulo pouco visível.

Rostro longo, aproximadamente igual ao comprimento do restante da carapaça, formado por dois espinhos contíguos por cerca de metade do comprimento, separados na porção distal, onde podem divergir, ou continuar quase paralelos.

Olhos sem órbitas; espinhos pré e pós-oculares agudos.

Antenas visíveis dorsalmente; artículo basal estreito, com espinho no ângulo ântero-externo.

Quelípedes mais longos que a carapaça; mero subcilíndrico, com uma fileira de quatro espinhos na superfície externa; palma sem espinhos, alongada; dedos curtos, fortes, com hiato na porção basal, maior na fêmea que no macho.

Patas ambulatórias pubescentes; primeiros dois pares longos, delgados; terceiro e quarto pares mais curtos, preênseis, com dáctilos espinhosos no bordo interno; terceiro par o mais curto, mero com geralmente dois espinhos fortes em forma de gancho na sua margem interior; quarto par maior que o terceiro, com própodo quase retilíneo, espessado distalmente, dáctilo mais curto que o própodo e menos encurvado que o do terceiro par.

Dimensões.- fêmea com até $30 \mathrm{~mm}$ de comprimento, incluindo o rostro.

Coloração.- Amarelo-alaranjado claro, com palmas mais escuras (RATHBUN, 1925).

Ecologia.- Conhecido de 55 a 686 metros de profundidade, mais comumente em profundidades superiores a 180 metros, em vários tipos de fundo. $\mathrm{O}$ material examinado foi coletado entre 173 e 200 metros de profundidade, em substrato de lama arenosa e organogênico; temperatura de 15,0 a $18,7^{\circ} \mathrm{C}$ e salinidade de 35,7 a $36,0 \%$ o. A espécie foi encontrada fazendo parte de povoamentos batiais em águas temperadas quentes.

Comentários.- Embora haja um certo número de diferenças entre o material examinado e as descrições dos autores, foi preferido considerar como pertencente à espécie já conhecida, A. furcillatus. As principais diferenças são: a) número de espinhos em cada região branquial (dois no material de A. MILNE EDWARDS \& BOUVIER, 1923, e apenas um no presente material); b) tamanho da elevação sobre os lobos hepáticos (bem nítida nas figuras dos autores, pouco visível no material examinado); c) número de espinhos no mero da terceira pata ambulatória (os autores sempre se referem a três, porém o material apresenta apenas dois espinhos, exceto um 
espécimen, com dois espinhos no lado direito e quatro no lado esquerdo). Quanto à porção livre dos espinhos do rostro, sensivelmente paralela, corresponde à figura $6 \mathrm{da}$ pl. 10 de A. MILNE EDWARDS \& BOUVIER (1923).

Gênero Eucinetops Stimpson, 1860.

Eucinetops STIMPSON, 1860:191.- A. MILNE EDWARDS, 1875:119.RATHBUN, 1925:80.- GARTH, 1958:51.

Redescriçãao.- Carapaça oblonga. Rostro pequeno, pouco defletido. Órbitas pequenas, ncluindo apenas a base dos pedúnculos oculares; ângulo externo agudo, espiniforme; margem superior com uma fissura, porém desprovida de dentes ou espinhos. Fosseta antenular arredondada, margem obtusa. Artículo basal das antenas pequeno, com um dente ou espinho no ângulo ântero-externo; porção móvel com os dois primeiros artículos muito largos. Epístoma muito curto ou ausente. Cavidade bucal muito larga anteriormente.

Mero dos maxilípedes externos triangular ou subtriangular, ângulo ânteroexterno elevado, margem distal mais longa; palpo muito curto e grosseiro, artículo terminal muito mais estreito que os demais.

Espécie-tipo.- Eucinetops lucasii Stimpson, 1860.

Distribuiçãa geográfica.- Uma espécie no Atlântico Ocidental, E. blackiana Rathbun, e três outras no Pacífico Oriental, desde o México até o Equador: E. lucasii Stimpson, E. rubellula Rathbun e E. panamensis Rathbun.

Eucinetops blackiana Rathbun, 1896.

Eucinetops blackiana RATHBUN, 1896:141; 1925:88, pl. 23, figs. 1-2.

Localidade tipo.- Port Royal, Jamaica.

Outros registros.- BAAMAS (RATHBUN, 1925); JAMAICA (RATHBUN, 1925); PORTO RICO (RATHBUN, 1925); BONAIRE (RATHBUN, 1925, 1936).

Material examinado.- $1 \mathrm{~F}(\mathrm{OV})$ coletada na Playa Cañaveral, COLÔMBIA (SMF).

Distribuição geográfica.- Atlântico Ocidental: desde as Baamas até a Colômbia.

Redescrição.- Carapaça com superfície irregular; regiões medianas elevadas, regiões hepáticas abaixadas, separadas das branquiais por sulco profundo e por seio marginal. Margens ântero-laterais quase retilíneas, convergindo um pouco para a frente, armadas com tubérculos; ângulos póstero-laterais marcados por espínulos; alguns tubérculos na superfície superior das regiões branquiais e intestinal.

Rostro formado por dois lobos triangulares, arredondados, com um espinho curvado por cima no ápice e separados por seio em forma de $\mathrm{V}$.

Dente pós-ocular mais longo que largo, agudo, curvado para cima, separado da margem supra-ocular por um seio estreito e arredondado. Olhos com pedúnculo adelgaçando em direção à córnea, ápice um pouco dilatado. 
Primeiro artículo móvel das antenas com um lobo ântero-externo, com um dente no ângulo externo, não alcançando a extremidade do rostro.

Quelípedes pequenos, lisos, brilhantes; carpo com um tubérculo perto do mero; mão elevada, com as margens convergentes em direção aos dedos.

Patas ambulatórias subcilíndricas, dáctilos muito delgados, curvos.

Dimensões.- Até pouco mais de $6 \mathrm{~mm}$ de comprimento.

Ecologia.- Conhecida apenas de águas à pequena profundidade; o espécimen examinado foi encontrado entre 3 e 4 metros de profundidade, sob pedras. Pelos poucos dados disponíveis, deveria ser considerada espécie habitante de povoamentos de substratos duros e rasos em águas tropicais.

\section{REFERÊNCIAS BIBLIOGRÁFICAS}

ABELE, L. G.; KIM, W., 1986. An illustrated guide to the marine decapod crustaceans of Florida. Tallahassee: Florida State University Department of Biological Science (State of Florida Department of Environmental Regulation Technical Series, v. 8, n. 1, parts 1-2)

ALCOCK, A., 1898. Materials for a carcinological fauna of India. No. 1. The Brachyura Oxyrhyncha. J. As. Soc. Bengal, 64 (part II, No 2): 157-291.

BALSS, H., 1929. Decapoden der Rotten meeres. IV. Oxyrhyncha und schlussbetrachtungen. (Expedition S. M. Schiff "Pola" in das Rotte meer, Zool. Ergeb. 36). Denkschr. Akad. Wiss. Vien, 102: 1-30.

BALSS, H., 1957. Decapoda, part VIII: systematik. In: BRONN, H. B., Klassen und Ordnungen des tierreichs 5(1):7(12): 1505-1672. Leipzig, Akademische Verlags.

BARNARD, K. H., 1950. Descriptive catalogue of south african decapod Crustacea (crabs and shrimps). Ann. S. Afr. Mus., 38:1-837.

BELL, T., 1835. Some account of the Crustacea of the coasts of South America, with description of new genera and species. I. Oxyrhynchi. Proc. Zool. Soc. London, 3: 169-173.

BENNET, E. W., 1964. The marine fauna of New Zealand: Crustacea Brachyura. Dep. Sci. Industr. Res. Bull. 153 (N. Z. Oceanogr. Mem. 22). 120 pp.

BORRADAILE,L. A., 1916. Crustacea. I. Decapoda. Brit. Antarct. ("Terra Nova") Exp. 1910 Nat. Hist. Rep. Zool. 3: 75-126.

BOSCHI, E. E., 1964. Los crustaceos decapodos Brachyura del litoral bonaerense. Bul. Inst. Biol. Mar., 6: 1-100.

BOUVIER, E. L., 1940. Décapods marcheurs. Faune de France, 37. Paris: Paul Lechevalier et Fils. 404 p., 14 plates.

CAPART, A., 1951. Crustacés decapodes brachyures. Res. Sc. Exped. Oceanogr. Belge Atlant. Sud., 3(1): 11-205.

CHACE JR., F. A., 1940. Reports on the scientific results of the Atlantis Expeditions to the West Indies, under the joint auspices of the University of Havana and Harward University. The brachyuran crabs. Torreia, 5: 3-67.

COELHO, P. A., 1967/9. A distribuição dos crustáceos decápodos reptantes do Norte do Brasil. Trabs. Oceanogr. Univ. Fed. Pernambuco, 9/11: 223-238. 
COELHO, P. A., 1971. Nota prévia sobre os Majidae do Norte e do Nordeste do Brasil (Crustacea, Decapoda). Arq. Mus. Nac., 54: 137-143.

COELHO, P. A., 1972. Descrição de três espécies novas de Majidae do Brasil (Decapoda, Brachyura). Trabs. Oceanogr. Univ. Fed. Pernambuco, 13: 119-132 [distribuido 1973].

COELHO, P. A. \& RAMOS, M. A., 1972. A constituição e a distribuição da fauna de decápodos do litoral leste da América do Sul entre as latitudes de $5^{\circ} \mathrm{N}$ e $390 \mathrm{oS}$. Trabs. Oceanogr. Univ. Fed. Pernambuco, 13: 133-236 [distribuido 1973].

CUNNINGHAM, R. O., 1871. Notes on Reptiles, Amphibia, Fishes, Molluscs and Crustacea obtained during the voyage of H. M. S. "Nassau" in the years 1866-69. Trans. Linn. Soc. London, 27: 465-502.

DANA, J. D., 1851. On the classification of the majoid Crustacea or Oxyrhyncha. Am. J. Sci., Ser. 2, 11: 425-434.

DANA, J. D., 1852/3. Crustacea. Philadelphia: C. Shermanm 1852/3. 2v. 1816pp. (United States Exploring Expedition during the years 1836, 1839, 1840, 1841, 1842 under Charles Wilkes, U. S. N., 13).

DOFLEIN, F.; BALSS, H., 1912. Die Dekapoden und Stomatopoden der Hamburger Magalhaensischen Sammelreise 1892/93. Mitt. Nat.-Hist. Mus., 29:25-44.

DRACH, P. \& GUINOT, D., 1982. Les Inachoididae Dana, famille de Majoidea caracterisée par des connexions morphologiques d'un type nouveau entre carapace, pleurites, sternites et pléon (Crustacea, Decapoda). C. R. Acad. Sci. Paris, Sér. III, 297: 37-42.

GARTH, J. S., 1957. Reports of the Lund University Chile Expedition 1948-49. 29. The Crustacea Decapoda of Chile. Lunds Univ. Arss., Avd. 2, 53(7): 3-131.

GARTH, J. S., 1958. Brachyura of the Pacific coast of America, Oxyrhyncha. Allan Hancock Pac. Exped., 21(1): 1-499; 21(2): 500-854.

GRIFFIN, D. J. G., 1966a. The marine fauna of New Zealand: spider crabs, family Majidae (Crustacea, Brachyura). Bull. N. Z. Dep. Sci. Ind. Res. (Mem. N. Z. Oceanogr. Inst., 35), 172: 1-111.

GRIFFIN, D. J. B., 1966b. A review of the australian majid spider crabs (Crustacea. Brachyura). Aust. Zool., 13: 259-298.

GRIFFIN, D. J. B. \& TRANTER, H. A., 1986. The Decapoda Brachyura of the Siboga Expedition. Part VIII. Majidae. Siboga-Expeditie, monogr. 39, C4 (=Livraison 148):1-335.

GUÉRIN, M. F. E., 1825. Triangulaires. Pages 697-701, in: LATREILLE, P. A. Encyclopedie méthodique: entomologie ou histoire naturelle des Crustacés, des Arachnides et des Insects. Paris.

GUÉRIN, M. F. E., 1828. Mémoire sur 1’Eurypode, nouveau genre du crustacé décapode brachyure. Mem. Mus. Hist. Nat. Paris, 16: 345-356.

GUINOT, D., 1966. La faune carcinologique (Crustacea Brachyura) de l'Océan Indien Ocidental et de la Mer Rouge. Catalogue, rémarques biogeographiques et bibliographie. In: Réunion de spécialistes C. S. A. sur les crustacés, Zanzibar, 1964. Mem. IFAN, 77:237-352. 
GUINOT, D., 1978. Principes d'une classification évolutive des crustacés décapodes brachyoures. Bull. Biol. Fr. Belg., 112: 211-290.

GUINOT, D., 1984. Le genre Leurocyclus Rathbun, 1897 (Crustacea Decapoda Brachyura). Bull. Mus. Natn. Hist. nat., 4e sér., 6A(2):377-395.

GUINOT, D.; RICHER DE FORGES, B. Affinités entre les Hymenosomatidae MacLeay, 1838 et les Inachoididae Dana, 1851 (Crustacea, Decapoda, Brachyura). Zoosystema, 19(2-3):453-502, 1997.

LAGERBERG, T., 1905. Anomura und Brachyura der schwedischen sudpolarexpedition Wissenchaftlich Ergebenisse der Swedischen Sudpolar-Expedition 19011903, 5(Zool.): 1-39.

LEMOS DE CASTRO, A., 1954. Fauna do Distrito Federal. IX. Descrição de uma nova espécie do gênero Eucinetops Stimpson (Decapoda: Majidae). Rev. bras. Biol., 13(4):355-358.

LENZ, M., 1902. Die Crustaceen der Sammlung Plate (Decapoda und Stomatopoda). Zool. Jahrb. Jena, 5 (Suppl.): 731-732.

MACLEAY, W. S., 1838. On the brachyourous decapod Crustacea brought from the Cape by Dr. Smith, in: Illustrations of Annulosa of South Africa, etc., 75 pp.

MELO, G. A. S., 1990. A presença, no litoral sudeste brasileiro, de espécies de Brachyura (Crustacea: decapoda) originárias das regiões biogeográficas Magelânica e Argentina do Atlântico Sul. Atlântica, 12(2):71-83, 1990.

MELO, G. A. S., 1996. Manual de identificação dos Brachyura (caranguejos e siris) do litoral brasileiro. São Paulo: Plêiade/FAPESP, 1996.

MIERS, E. J., 1879. On the classification of the majoid Crustacea or Oxyrhyncha, with a synopsis of the families, subfamilies and genera. J. Lin. Soc. London, Zool., 14:634-673

MIERS, E. J., 1886. Report on the brachyura collected by H. M. S. Challenger during the years of 1873/1876. Challenger Rep., Zool., 17: 1-361.

MILNE-EDWARDS, A., 1873/81. Études sur les Xiphosures et les Crustacés de la région méxicaine. Miss. Sci. Méxique. Rech. Zool., 5: 1-368.

MILNE-EDWARDS, A. \& BOUVIER, E. L.., 1923. Reports on the results of dredging under the supervision of Alexander Agassiz in the Gulf of Mexico (18771878), in the Caribbean Sea (1878-1879) and along the atlantic coast of the United States (1880), by the U. S. Coast Survey Steamer "Blake". 42. Les Porcellanides et des Brachyures. Mem. Mus. Comp. Zool., 27: 1-127.

MONOD, T., 1956. Hippidea et Brachyura ouest-africains. Mem. IFAN, 45: 1-674.

POWERS, L. W., 1977. A catalogue and bibliography to the crabs (Brachyura) of the Gulf of Mexico. Contrib. Mar. Sci. Port Aransas Mar. Lab., 20: 1-190.

RATHBUN, M. J.., 1894. Notes on crabs of the family Inachidae in the U. S. National Museum. Proc. U. S. Nat. Mus., 17: 43-75.

RATHBUN, M. J., 1896. Description of a new genus and four new species of crabs from the West Indies. Proc. U. S. Nat. Mus., 19: 141-144.

RATHBUN, M. J., 1987a. A revision of the nomenclature of the Brachyura. Proc. Biol. Soc. Washington, 11:153-167. 
RATHBUN, M. J., 1897b. List of decapod Crustacea of Jamaica. Ann. Inst. Jamaica, 1: $1-46$.

RATHBUN, M. J., 1898. The Brachyura collected by the U. S. Fish Commision Steamer Albatross on the voyage from Norfolk, Virginia, to San Francisco, California, 1887-1888. Proc. U. S. Nat. Mus., 21: 567-616.

RATHBUN, M. J., 1925. The spider crabs of America. Bull. U. S. Nat. Mus., 129: 1613.

RATHBUN, M. J., 1936. Zooloogische ergebenisse einer Reise nach Bonaire, Curaçao und Aruba in Jahre 1930. 17. Brachyuran Crustacea from Bonaire, Curaçao and Aruba. Zool. Jahrb. Syst., 67: 379-388.

RODRÍGUEZ, G., 1980. Los crustaceos decápodos de Venezuela. Caracas: Instituto Venezolano de Investigaciones Científicas, 494 pp.

SAKAY, T., 1976. Crabs of Japan and the adjacent seas. Part I. Tokyo: Kodansha. 773 pp.

SAMOUELLE, G., 1819. The entomologist's usefull compendium, or an introduction to the knowledge of british insects. London, 1819.

SOTO, L. A., 1980. Decapd Crustacea shelf-fauna of the Northeastern Gulf of Mexico. An. Cent.Cien. Mar. Limnol. Univ. Nat. Auton. 7:79-110.

SOUZA, J. A. F. Brachyura da plataforma meridional do Rio Grande do Sul, Brasil (Crustacea, Decapoda). Nauplius, 5(2):33-58, 1997.

STEVCIC, Z., 1994. Contribution to the re-classification of the family Majidae. Per. Biol., 96(4):419-420.

STIMPSON, W., 1860. Notes on North American Crustacea, in the Museum of the Smithsonian Institution. No. II. Ann. Lyc. Nat. Hist. N. Y., 7: 176-246.

STIMPSON, W., 1871. Preliminary report on the Crustacea dredged in the Gulf Stream in the Streats of Florida, by L. P. de Pourtalès, Assist. U. S. Coast Survey. Part I. Brachyura. Bull. Mus. Comp. Zool., 2: 109-160.

WILLIAMS, A. B., 1965. Marine decapod crustaceans of the Carolinas. Fish. Bull., 65: 1-298.

WILLIAMS, A.B., 1984. Shrimps, lobsters, and crabs of the atlantic coast of eastern United States, Maine to Florida. Washington: Smithsonian Institution Press. 550 pp. 


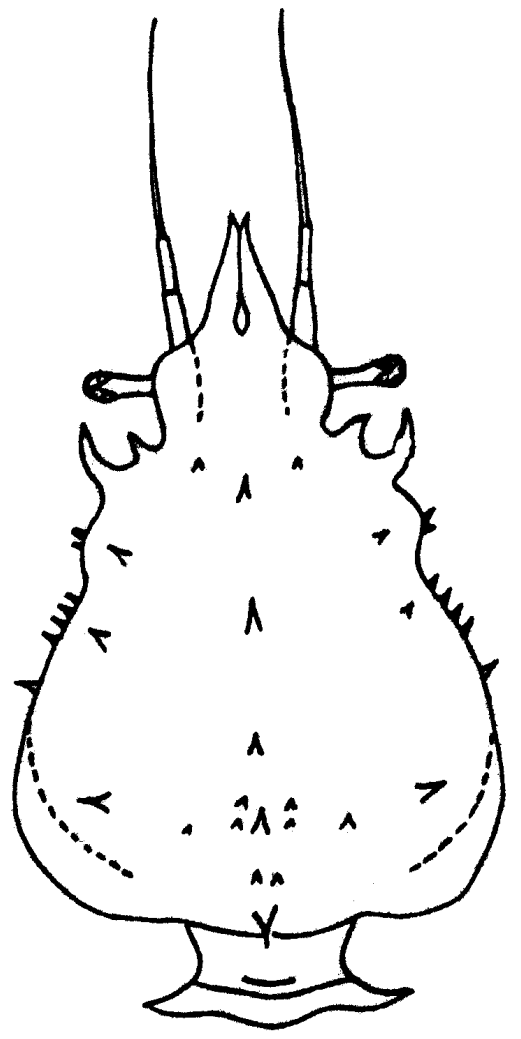

Fig. 1 - Eurypodius latreillei Guérin: carapaça, vista dorsal. O exemplar mede 14,5 mm de comprimento e $9,8 \mathrm{~mm}$ de largura da carapaça; a escala representa $1 \mathrm{~mm}$. 

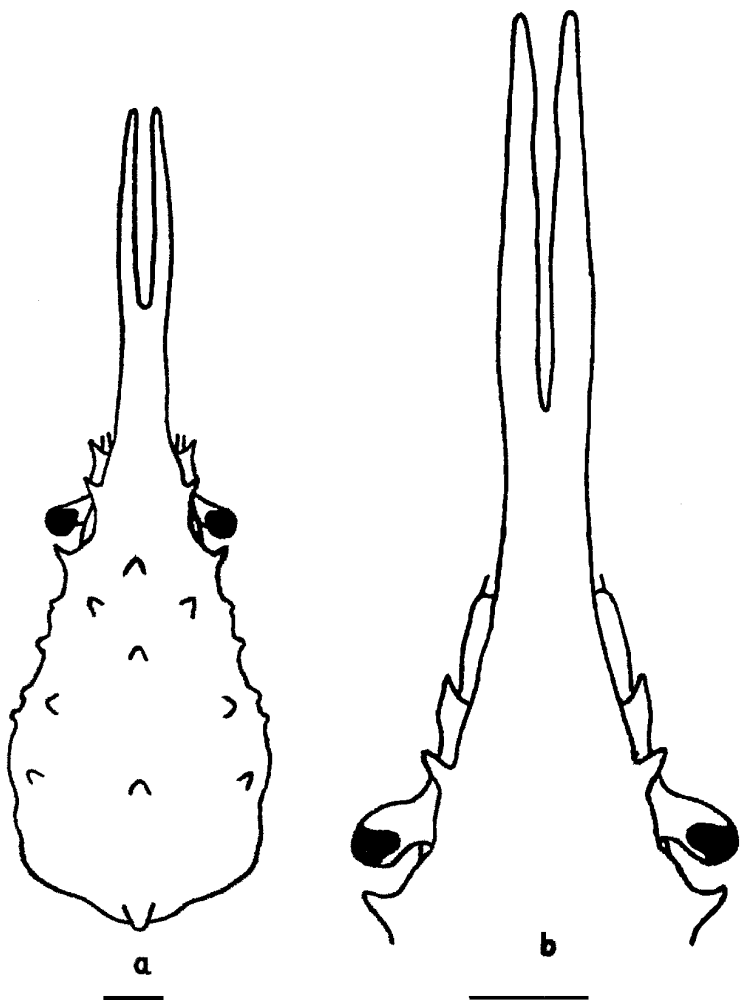

Fig. 2 - Anomalothir furcillatus (Stimpson). a) carapaça, vista dorsal; b) porção anterior, vista dorsal. 0 exemplar mede $14,3 \mathrm{~mm}$ de comprimento e $4,5 \mathrm{~mm}$ de largura da carapaça; a escala representa $1 \mathrm{~mm}$. 


\section{ANEXO}

LISTA DAS ESTAÇÕES REALIZADAS POR EXPEDIÇÕES OCEANOGRÁFICAS.

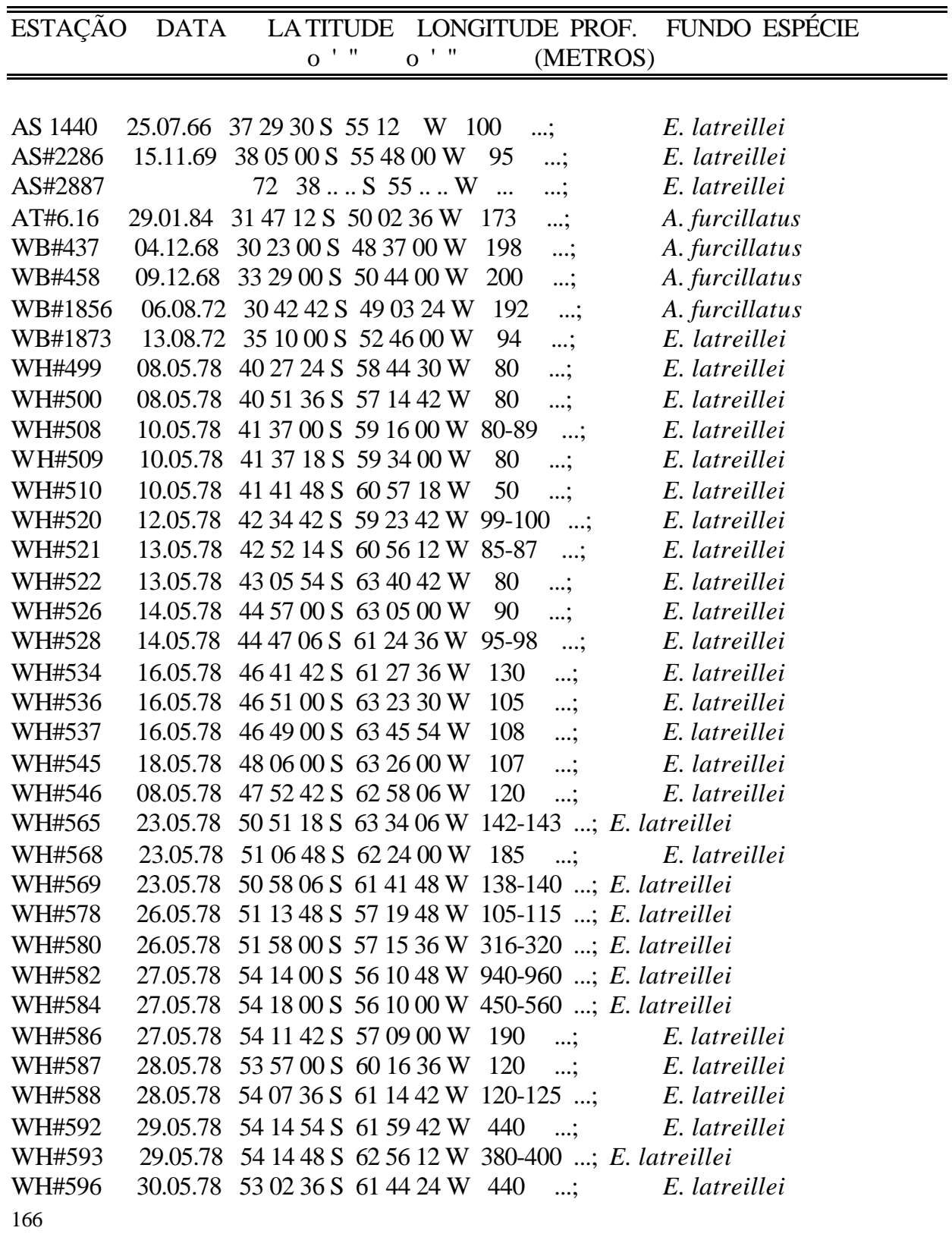




\begin{tabular}{|c|c|c|c|c|}
\hline H600 & 31.05 .78 & $535906 \mathrm{~S} 655142 \mathrm{~W}$ & 80 & E. latreillei \\
\hline VH\#603 & 6.06 .78 & $32642 \mathrm{~S} 661524 \mathrm{~W}$ & 50 & E. latreillei \\
\hline /H\#604 & 5.06 .78 & $0000 \mathrm{~S} 664336 \mathrm{~W}$ & $86-88 \quad \ldots ;$ & E. lat \\
\hline H\#606 & 6.78 & S 66 & $95-107 \ldots$ & E. lat \\
\hline H\#607 & & & $15 \ldots ;$ & E. latreillei \\
\hline H\#609 & .78 & S & $60 \ldots ;$ & E. latreillei \\
\hline H\#613 & & & $300 \ldots$ & E. latreillei \\
\hline H\#614 & & & 165 & E. latreillei \\
\hline H\#615 & 09.06 .78 & $512748 \mathrm{~S} 634342 \mathrm{~W}$ & 150 & E. latreillei \\
\hline & & & 148 & \\
\hline \#617 & 78 & & 130-142 ...; & E. latreillei \\
\hline & .78 & & 130 & E. latre \\
\hline 19 & & & $125 \ldots$ & atreillei \\
\hline H\#620 & 10. & 51 & 90 & E. latre \\
\hline & & $N$ & 90 & E. la \\
\hline 23 & & 502 & $84-85 \quad \ldots ;$ & E. la \\
\hline \#626 & 78 & 50 & 120 & E. la \\
\hline & & & 120 & E. la \\
\hline \#628 & 78 & 49 & 140 & E. lat \\
\hline & & & $141-145$ & latreillei \\
\hline & & & 145 & E. latreill \\
\hline H\#631 & 12. & 50 & 160 & E. la \\
\hline$\neq 632$ & 78 & 50 & 160 & E. la \\
\hline I\#633 & 78 & 49 & $160-165 \ldots$ & treillei \\
\hline 39 & 78 & 49 & 170 & E. latreillei \\
\hline & & & 160 & E. la \\
\hline & & 48 & $190-195 \ldots$ & E. la \\
\hline I\#642 & 78 & 48 & 130 & E. la \\
\hline & & & 116 & E. la \\
\hline & & W & 100 & E. la \\
\hline & & & 95 & E. la \\
\hline & & 46 & 105 & E. la \\
\hline 54 & 78 & 47 & 130 & E. latreillei \\
\hline I\#655 & & & $140-143$ & treillei \\
\hline \#656 & & & $930-950$ & atreillei \\
\hline & & & 110 & E. latreill \\
\hline \#661 & & 45 & 100 & E. lat \\
\hline I\#662 & 78 & 44 & 100 & E. latreillei \\
\hline \#667 & & 44 & 85 & E. lat \\
\hline VH\#671 & 21. & S 6135 & 80 & E. latreillei \\
\hline \#673 & 21. & 44 & 100 & E. latreill \\
\hline & & $435448 \mathrm{~S} 593942 \mathrm{~W}$ & $810-920$ & treillei \\
\hline NH\#675 & 22.06 .78 & 593 & $680-640$ & latreillei \\
\hline NH\#678 & 23.06 .78 & $415706 \mathrm{~S} 590618 \mathrm{~W}$ & 85 & E. latre \\
\hline
\end{tabular}

Trab. Oceanog. Univ. Fed. PE, Recife, 27(1):149-168, 1999. 


$\begin{array}{lrlllll}\text { WH\#679 } & 23.06 .78 & 415724 \mathrm{~S} & 594448 \mathrm{~W} & 80 & \ldots ; & \text { E. latreillei } \\ \text { WH\#681 } & 23.06 .78 & 423200 \mathrm{~S} & 604500 \mathrm{~W} & 80 & \ldots ; & \text { E. latreillei } \\ \text { WH\#682 } & 23.06 .78 & 423948 \mathrm{~S} & 613048 \mathrm{~W} & 78-80 & \ldots ; & \text { E. latreillei } \\ \text { WH\#683 } & 24.06 .78 & 412706 \mathrm{~S} & 633630 \mathrm{~W} & 47 & \ldots ; & \text { E. latreillei } \\ \text { WH\#684 } & 24.06 .78 & 412742 \mathrm{~S} & 622536 \mathrm{~W} & 38-40 & \ldots ; & \text { E. latreillei } \\ \text { WH\#690 } & 25.06 .78 & 402200 \mathrm{~S} & 591442 \mathrm{~W} & 70-72 & \ldots ; & \text { E. latreillei } \\ \text { WH\#691 } & 25.06 .78 & 402718 \mathrm{~S} 581230 \mathrm{~W} & 85 & \ldots ; & \text { E. latreillei } \\ \text { WH\#693 } & 26.06 .78 & 402354 \mathrm{~S} 560712 \mathrm{~W} & 940-1040 \ldots ; \text { E. latreillei } \\ \text { WH\#696 } & 27.06 .78 & 403318 \mathrm{~S} 563848 \mathrm{~W} & 300-310 & \ldots ; & \text { E. latreillei } \\ \text { WH\#697 } & 27.06 .78 & 402748 \mathrm{~S} 566148 \mathrm{~W} & 164 & \ldots ; & \text { E. latreillei }\end{array}$

\section{LISTA DAS COLETAS NÃO FAZENDO PARTE DE EXPEDIÇÕES OCEANOGRÁFICAS}

\section{COLÔMBIA:}

Playa Cañaveral, 12.10.1978, 3-4 m, sob pedras. E. blackiana ARGENTINA:

Ostpatagonische Bank, $44^{\circ} 14^{\prime} \mathrm{S}, 61^{\circ} 23^{\prime} \mathrm{W}, 27.08 .1887,110 \mathrm{~m}$; E. latreillei

Ostpatagonische Bank, $45^{\circ} \mathrm{S}, 15.06 .1906,110 \mathrm{~m}$; E. latreillei

Ostpatagonische Bank, 1912; E. latreillei

Comodoro Rivadavia, 02.1916; E. latreillei

Puerto Deseado, 17.03.1971; E. latreillei

Sem nome de localidade, $51^{\circ} 30^{\prime} \mathrm{S}, 65^{\circ} 00^{\prime} \mathrm{W}, 05.02 .1913,140 \mathrm{~m} ;$ E. latreillei

Sarmiento Bank, Terra do Fogo, 52 $24^{\prime} \mathrm{S}, 68^{\circ} 09^{\prime} \mathrm{W}, 13.08 .1910,47 \mathrm{~m}$; E. latreillei

Port Stanley, Ilhas Malvinas, 1895; E. latreillei

Port Williams, Ilhas Malvinas, 28.10.1893. E. latreillei 